\title{
Lower genital tract infections in infertile Nigerian women compared with controls
}

\author{
F E Okonofua, K A Ako-Nai, M D Dighitoghi
}

\begin{abstract}
Objective-To investigate the possibility that infertile Nigerian women have a higher rate of cervical colonisation with pathogenic and facultative organisms than fertile controls.

Design-The prevalence of common microorganisms in the vagina and endocervical canals of infertile women was compared with that of pregnant controls. Setting-The Obafemi Awolowo University Hospital Maternity Centre.

Subjects-92 infertile women were compared with 86 pregnant controls.

Main Outcome Measures-rates of isolation of Neisseria gonorrhoeae, Candida albicans, Trichomonas vaginalis and other facultative organisms in cases and controls.
\end{abstract}

Results-The rate of isolation of Neisseria gonorrheae was $17 \cdot 4 \%$ among infertile women compared with $10.5 \%$ in the group of pregnant women $(p>0.05)$. There was no significant difference between the groups in the rate of isolation of Candida albicans, Trichomonas vaginalis and other facultative organisms. High rates of isolation of microorganisms were observed in both groups. However, women with secondary infertility had higher rate of carriage of Neisseria gonorrheae, Candida albicans and Staphylococcus aureus as compared with women with primary infertility. Nearly $15 \%$ of infertile women had previous episodes of pelvic inflammatory disease and $26 \%$ had had induced abortions. A positive history of vaginal discharge was a poor predictor of vagina and endocervical carriage of microorganisms.

Conclusions-High rates of pathogenic organisms exist in the lower genital tract of infertile women and controls. Women with secondary infertility are more likely to have pathogenic organisms than women with primary infertility. A policy of routinely screening women for lower genital tract infections should be pursued in this population because of the high rate of infection.

(Genitourin Med 1995;71:163-168)

Keywords: Infertility; genital tract infection

Introduction

Infertility is currently regarded as a major public health problem in Nigeria. In some rural parts of Nigeria, approximately $30 \%$ of couples may have difficulties in achieving a desired conception. ${ }^{1}$ Available evidence suggests that the majority of the cases of infertility in the country are due to previous reproductive tract infections that produce severe damage to the female pelvic organs. It has been estimated that about two-thirds of the cases of infertility in Nigeria are attributable to infections. ${ }^{2}$ The laparoscopic investigation of infertility in Nigerian hospitals has demonstrated the presence of pelvic infection and bilateral tubal occlusion in $35 \%$ of infertile women in Ibadan, ${ }^{3} 44 \%$ in Ile-Ife ${ }^{4}$ and $65 \%$ of women in Jos. ${ }^{5}$

Although data are presently limited, it is believed that the majority of the infections and subsequent pelvic damage are produced by sexually transmitted diseases (STDs) particularly Neisseria gonorrhoeae, Chlamydia trachomatis and bacterial vaginosis. ${ }^{6}$ However, clinical and epidemiological evidence suggests that post-abortal and postpartum infections may be equally important. ${ }^{789}$

To demonstrate the relative importance of the various types of sexually transmitted diseases in producing infertility at the community level would require either serosurveys or culture-based studies. Serosurveys are an excellent method for determining the levels of STD transmission in areas of high endemicity but they are difficult to interpret because of the cross-reacting nature of serologic tests for antibodies to Neisseria gonorroheae, Hemophilus ducreyi, Chlamydia trachomatis and herpes simplex type 2. By contrast, culture based studies are accurate but they suffer from the disadvantage that they cannot be used to infer previous infections that produced tubal damage in the first place and would have to be prospective to be able to demonstrate meaningfully a link between pelvic infection and infertility. However, culture-based studies are useful in determining ongoing levels of infection and can be used to answer a number of relevant questions on the relationship between infections and infertility.

A particularly pertinent question is: are infertile women at risk of developing lower genital tract infections? Theoretically, it would be expected that infertile women would have higher levels of lower genital infections than fertile women since a number of the infections can be carried for years in the lower genital tract. It is also likely that infertile women may be more susceptible to re-infection with the same or another pathogenic organism than fertile women because of continuation of previous sexual behaviour that 
put them at risk in the first place. This study was designed to investigate the possibility that infertile Nigerian women have higher levels of lower genital tract infections than fertile women and the types of organisms that are most commonly implicated. Specifically, the paper describes the aerobic and facultative anaerobic micro-organisms colonising the endocervices of fertile and infertile women and the antibiotic sensitivity of some of the isolates. The results should enable us to determine whether a policy of routinely screening these women in the gynaecological clinics is justifiable and to comment on the role of infection in infertility.

\section{Subjects and methods}

Subjects The study was carried out at the Obafemi Awolowo University Hospital, Nigeria between 20 September 1990 and 16 December 1991 and consisted of patients and controls. The patients were 92 infertile women attending the infertility and endocrinology clinic of the hospital during the period. Infertility was defined according to the WHO current definition of infertility, that is, the inability to establish a pregnancy within two years for couples of reproductive age who are having intercourse without contraception. ${ }^{10}$ Primary infertility refers to those who have never been pregnant while secondary infertility refers to those who have had at least one proven pregnancy (including an abortion). The controls were 86 randomly selected pregnant women who attended the Wednesday antenatal clinic of the hospital. The pregnant women were selected from among those attending the antenatal clinic during the same period as the cases of infertility. To ensure comparability of cases and controls, we selected the pregnant women from among women of the same age and educational level as the cases of infertility. This is because we recognised that age and educational level are the two factors most likely to affect the rates of sexually transmitted diseases in the community. Thus, after first identifying an infertile woman, all pregnant women of the same age and educational level were identified in the ensuing antenatal clinic. One of these women would then be randomly selected by balloting for inclusion into the study. Using this procedure, eligible controls could not be found for six women from the immediate antenatal clinics, thereby limiting the study to 92 cases and 86 controls. The study was carefully explained to both groups of subjects and they were assured of strict confidentiality of information obtained. All recruited women agreed to participate in the fully explained protocol and were included in the study.

At the onset, the women were assisted in completing a structured questionnaire which sought information on their socio-demographic characteristics, their previous pregnancy and infertility history, history of previous treatment of sexually transmitted diseases and vaginal discharge and reproduc- tive history including previous use of contraception and abortion. The infertile women were fully investigated with hysterosalpingography, laparoscopy, semen analysis and endometrial biopsy and the probable cause of infertility ascertained.

The infertile women had a duration of infertility ranging between 2 and 20 years with a median duration of 6 years. Twenty four $(26.1 \%)$ of the infertile women had primary infertility while $68(73.9 \%)$ had secondary infertility. After full infertility investigations; 15 women were found to have bilateral tubal occlusion; seven had unilateral tubal occlusion and pelvic adhesions; six had uterine fibroids; 16 had semen abnormalities; 18 had ovulatory and menstrual problems; four had problems in both partners; while no cause could be found in 26 couples.

Collection and processing of samples In both groups of women, a sterile bivalve speculum examination was carried out by the attending obstetrician and gynaecologist and two endocervical and one high vaginal samples taken with the aid of two sterile cotton-tipped applicators (Evepon Industries Ltd., Nigeria). The swab stick was rotated several times along and around the walls of the endocervix. A wet mount of each sample was prepared by addition of normal saline to the sample on a clean glass slide. The wet mount was then examined under the microscope with $\times 100$ for the presence of white blood cells, red blood cells, Candida albicans, Trichomonas vaginalis, bacteria and epithelial cells.

Primary isolation for possible anaerobic organisms was performed by inoculating the first swab on freshly prepared cooked meat medium, brain heart infusion broth (BHI) and thioglycolate broth. The fluid media were incubated anaerobically and after growth was observed, each tube was subcultured onto various anaerobic plates. For primary isolation of aerobic organisms, each endocervical swab was streaked onto blood agar, ThayerMartin agar, mannitol salt agar (MSA), eosin methylene blue agar and sabouraud dextrose agar supplemented with penicillin/neomycin.

All plates were subsequently incubated at $37^{\circ} \mathrm{C}$ for a minimum of five days. All plates were examined daily for growth and based on the gram stain reaction and colonial morphology, each discrete colony was subjected to further characterisation (cultural and biochemical) using standard microbiological techniques (Finegold et al 1978). For example, gram-negative short rods suspected to be enteric organisms were confirmed by the use of the API $10 \mathrm{~S}$ system (La Balme Les Grotes, 38390 Montalieu Vercieu, France) and Staphylococcus aureus was speciated using coagulase tube test on pooled human plasma. Fungal specimens were inoculated into Sabouraud dextrose agar supplemented with penicillin/neomycin and incubated initially at $29^{\circ} \mathrm{C}$ for 3-7 days and speciation as Candida albicans was done by germ tube production on corn meal agar. Chlamydospore production in nutritionally deficient media and trypan blue agar was also used for identification of the 
fungus. Neisseria species were identified initially on Thayer-Martin agar, and subsequently with fermentation of CTA sugars and oxidase test.

Antibiotic sensitivity test was performed using the agar diffusion method described by Ericsson and Sherris (1971). Commercially prepared antibiotics (AB Biodisk Pyramidvagen Soln, Sweden) were used with the following concentrations: Ampicillin $10 \mu \mathrm{g}$, cefotaxime $30 \mu \mathrm{g}$, streptomycin $30 \mu \mathrm{g}$, trimethroprim $5 \mu \mathrm{g}$, sulphamethoxazole $223 \cdot 8$ $\mu \mathrm{g}+$ trimethoprim $1 \cdot 2 \mu \mathrm{g}, 25 \mu \mathrm{g}$, nalidixic acid $30 \mu \mathrm{g}$ and sulfisomidine $30 \mu \mathrm{g}$. Staphylococcus aureus ATCC 25923 and Enterobacter aerogenes ATCC 13042 were run as control organisms.

Table 1 Sociodemographic and clinical profiles of cases and controls

\begin{tabular}{|c|c|c|c|}
\hline & $\begin{array}{l}\text { Infertile women } \\
n=92\end{array}$ & $\begin{array}{l}\text { Pregnant women } \\
n=86\end{array}$ & n Odd ratios (CI) \\
\hline $\begin{array}{l}\text { Mean Age (range) years } \\
\text { Education }\end{array}$ & $27 \cdot 5(16-47)$ & $28 \cdot 0(16-48)$ & \\
\hline $\begin{array}{l}\text { None } \\
\text { Primary } \\
\text { Secondary } \\
\text { Tertiary } \\
\text { Parity, median (range) }\end{array}$ & $\begin{array}{c}17(18 \cdot 5) \\
39(42 \cdot 4) \\
30(32 \cdot 6) \\
6(6 \cdot 5) \\
1(0-3)\end{array}$ & $\begin{array}{l}15(17 \cdot 4) \\
37(43 \cdot 0) \\
28(32 \cdot 6) \\
6(7 \cdot 0) \\
4(0-12)\end{array}$ & \\
\hline $\begin{array}{l}\text { Per cent using } \\
\text { contraceptives }\end{array}$ & $3(3 \cdot 3)$ & $19(11 \cdot 6)$ & $0.2(0.05-0.9) \dagger$ \\
\hline previous induced abortions & $24(26 \cdot 1)$ & $8(9 \cdot 3)$ & $3.6(1.5-8 \cdot 8)^{\star}$ \\
\hline $\begin{array}{l}\text { of vaginal discharge } \\
\text { History of previous pelvic }\end{array}$ & $21(22 \cdot 8)$ & $17(19 \cdot 8)$ & $1 \cdot 2(0 \cdot 6-2 \cdot 4)$ \\
\hline inflammatory disease & $14(15 \cdot 2)$ & $2(2 \cdot 3)$ & $8.7(1.8-56.4) \dagger$ \\
\hline
\end{tabular}

CI-Confidence intervals; ${ }^{\star} \mathrm{p}<0.01 ; \mathrm{tp}<0.001$.

Table 2 Rate of isolation of pathogenic organisms from cases (infertile women) and controls (pregnant women)

\begin{tabular}{llcl}
\hline Isolate & $\begin{array}{l}\text { Infertile women } \\
\text { No }(\%)\end{array}$ & $\begin{array}{l}\text { Pregnant women } \\
\text { No }(\%)\end{array}$ & $P^{*}$ \\
\hline $\mathrm{n}$ & 92 & 86 & \\
Pathogenic aerobes & $16(17 \cdot 4)$ & $9(10 \cdot 5)$ & $\mathrm{ns}$ \\
$\begin{array}{l}\text { Neisseria gonorrhoeae } \\
\text { Staphylococcus aureus }\end{array}$ & $20(21 \cdot 7)$ & $10(11 \cdot 6)$ & $\mathrm{ns}$ \\
$\begin{array}{l}\text { Candida sp. } \\
\text { Trichomonas vaginalis }\end{array}$ & $23(25 \cdot 0)$ & $25(29 \cdot 1)$ & $\mathrm{ns}$ \\
Pathogenic Anaerobes & $4(4 \cdot 3)$ & $2(2 \cdot 3)$ & $\mathrm{ns}$ \\
$\begin{array}{l}\text { Clostridium sp. } \\
\text { Streptococcus sp }\end{array}$ & $3(3 \cdot 3)$ & $7(8 \cdot 1)$ & $\mathrm{ns}$ \\
(Beta haemolytic) & $7(7 \cdot 6)$ & $10(11 \cdot 6)$ & $\mathrm{ns}$ \\
$\begin{array}{l}\text { Opportunists } \\
\text { Pseudomonas aeruginosa }\end{array}$ & $3(3 \cdot 3)$ & $2(2 \cdot 3)$ & $\mathrm{ns}$ \\
Bacillus sp. & $5(5 \cdot 4)$ & $10(11 \cdot 6)$ & $\mathrm{ns}$ \\
Proteus vulgaris & $3(3 \cdot 3)$ & $2(2 \cdot 3)$ & $\mathrm{ns}$ \\
Escherichia coli & $2(2 \cdot 2)$ & $2(2 \cdot 3)$ & $\mathrm{ns}$ \\
\hline
\end{tabular}

ns = not significant.

${ }^{\star}$ Chi square test with Yates correction.

Table 3 Rate of microbial isolation in infertile women by type of infertility

\begin{tabular}{llll}
\hline & $\begin{array}{l}\text { Primary infertility } \\
n=24 \\
n(\%)\end{array}$ & $\begin{array}{l}\text { Secondary infertility } \\
n=68 \\
n(\%)\end{array}$ & P* \\
Isolate & $2(8 \cdot 3)$ & $14(20 \cdot 5)$ & $0 \cdot 02$ \\
\hline Pathogenic aerobes & $2(8 \cdot 3)$ & $18(26 \cdot 5)$ & $0 \cdot 001$ \\
Neisseria gonorrhoeae & $3(12 \cdot 5)$ & $20(29 \cdot 4)$ & $0 \cdot 01$ \\
Staphylococcus aureus & $1(4 \cdot 2)$ & $3(4 \cdot 4)$ & $\mathrm{ns}$ \\
Candida sp. & $1(4 \cdot 2)$ & $2(2 \cdot 9)$ & $\mathrm{ns}$ \\
Trichomonas vaginalis & $2(8 \cdot 3)$ & $6(8 \cdot 8)$ & $\mathrm{ns}$ \\
Pathogenic anaerobes & $1(4 \cdot 2)$ & $2(2 \cdot 9)$ & $\mathrm{ns}$ \\
Clostridium sp & $2(8 \cdot 3)$ & $3(4 \cdot 4)$ & $\mathrm{ns}$ \\
Opportunists & $1(4 \cdot 2)$ & $2(2 \cdot 9)$ & $\mathrm{ns}$ \\
Hemophilus sp. & $1(4 \cdot 2)$ & $3(1 \cdot 5)$ & $\mathrm{ns}$ \\
Pseudomonas aeruginosa & & & \\
Bacillus sp. & & & \\
Proteus vulgaris & Escherichia coli & &
\end{tabular}

ns-not significant.

$\star$-Chi square test with Yates correction.
Data analysis The frequency of isolation of the various pathogenic organisms was determined for the infertile and pregnant women and among the subgroups of causes of infertility. Any observed differences between the groups was tested for statistical significance with chi square test and Yates correction as appropriate.

\section{Results}

The sociodemographic and clinical profiles of the infertile and pregnant women are shown in Table 1. As indicated, both cases and controls had identical mean age and educational level. The pregnant women were more parous and reported higher "ever use" of contraceptives as compared with the infertile women. By contrast, a higher proportion of the infertile women reported previous pregnancy termination. A high proportion of both cases and controls $(22 \cdot 8 \%$ vs. $19 \cdot 8 \%)$ admitted experiencing vaginal discharge at the time of the study. However, as shown in table 1, more infertile women as compared with the controls had had symptomatic pelvic inflammatory disease (PID) in the past which required treatment $(p<0.002)$.

Multiple microbial isolates were cultured from the endocervices of the infertile and pregnant women. These included 203 isolates cultured from the endocervix of the 86 infertile women and 164 isolates recovered from the 92 pregnant women. There was apparently no difference in rate of isolation of organisms between pregnant and infertile women. As shown in table 2 , there was also no difference between pregnant and infertile women in the species of organisms isolated. Candida albicans was the commonest pathogenic organism isolated in both groups, with $25 \%$ of infertile women and $29.1 \%$ pregnant women harbouring the organism in their upper vagina and endocervix ( $p>0.05)$. Similarly, there was also no significant difference between infertile and pregnant women in the rate of isolation of Neisseria gonorrheae, Trichomonas vaginalis and Staphylococcus aureus. The rate of vaginal colonisation with pathogenic anaerobes and opportunistic organisms was also identical between pregnant and infertile women.

To determine whether the type of infertility has any effect on the pattern of vaginal and cervical colonisation by microorganisms, we compared the proportion of women with pathogenic organisms between those with primary infertility and those with secondary infertility. The results are presented in table 3. Women with secondary infertility had a higher rate of Neisseria gonorrheae $(20.5 \%)$ as compared with women with primary infertility $(8.3 \%) \quad(p<0.02)$. Similarly, secondarily infertile women had higher rates of colonisation with Candida albicans and Staphylococcus aureus. By contrast, there was no significant difference between the two groups of women in the rates of colonisation with Trichomonas vaginalis, Hemophilus $s p$ and other opportunistic vaginal organisms. 
Table 4 Antibiotic resistant pattern of isolated organisms

\begin{tabular}{|c|c|c|c|c|c|c|c|c|c|}
\hline \multirow[b]{2}{*}{ Bacterial isolates } & \multicolumn{9}{|c|}{$\%$ of resistant isolates } \\
\hline & $G M$ & $C T$ & $A M$ & $S C$ & $T S$ & $N A$ & $T R$ & $S U$ & $C L$ \\
\hline Neisseria gonorrhoeae & 0 & 100 & 100 & 100 & 100 & 0 & 50 & 100 & 100 \\
\hline Staphylococcus aureus & 50 & 33 & 75 & 75 & 33 & 42 & 33 & 50 & 58 \\
\hline Pseudomonas aeruginosa & 0 & 13 & 63 & 88 & 13 & 100 & 13 & 75 & 13 \\
\hline Hemophilus sp & 13 & 13 & 75 & 75 & 63 & 13 & 0 & 88 & 50 \\
\hline Bacillus & 17 & 67 & 67 & 83 & 0 & 33 & 50 & 67 & 33 \\
\hline Clostridium & 0 & 50 & 100 & 100 & 100 & 0 & 100 & 100 & 50 \\
\hline Escherichia coli & 50 & 0 & 100 & 50 & 50 & 0 & 50 & 50 & 50 \\
\hline Proteus vulgaris & 0 & 0 & 0 & 100 & 100 & 0 & 100 & 0 & 0 \\
\hline
\end{tabular}

$\mathrm{AM}=$ ampicillin $; \mathrm{CT}=$ cefotaxime $; \mathrm{CL}=$ chloramphenicol; $\mathrm{GM}=$ gentamicin $; \mathrm{NA}=$ nalidixic acid; $\mathrm{SC}=$ spectinomycin TS = sulfamethoxazole-trimethoprim; $S U$ = sufisomidine; $T R=$ trimethoprim

Further analysis showed that of the 16 infertile women from whom Neisseria gonorrheae was isolated, five had a past gynaecological history of pelvic inflammatory disease; four had bilateral tubal occlusion; and one had a previous history of chronic cervicitis. No associated risk factors could be identified in the other 10 infertile women from whom Neisseria gonorrheae was isolated.

To determine the predictive value of a history of vaginal discharge on the rate of isolation of a cervical pathogen among infertile women, we compared the isolates in women with vaginal discharge with those in women without vaginal discharge. Our results indicate that five isolates of Neisseria gonorrhoeae were made from the 38 infertile and pregnant women with vaginal discharge $(13.2 \%)$ compared with 20 isolates in the 140 women who had no history of vaginal discharge $(14 \cdot 3 \%)$. The difference was not statistically significant. There was also no significant difference in the rate of isolation of Candida albicans, Trichomonas vaginalis and Staphylococcus aureus between infertile women with vaginal discharge and those without vaginal discharge. Thus, a history of vaginal discharge did not appear to be a marker for the presence of the microorganisms in the vagina and endocervices of the women.

The antibiotic sensitivity pattern of the isolated organisms is presented in table 4. Neisseria gonorrheae, the most commonly isolated pathogenic organism, was uniformly sensitive to gentamicin and nalidixic acid and uniformly resistant to cefotaxime, ampicillin, spectinomycin and chloramphenicol. Similarly, Staphylococcus aureus, another pathogen, was resistant to many antibiotics and was only moderately sensitive to ampicillin, trimethroprim and sulfamethoxazoletrimethroprim.

\section{Discussion}

The study was designed to examine the association between asymptomatic cervical carriage of microorganisms and infertility in Nigerian women. We hypothesised that infertile women would be associated with a higher rate of cervical and vaginal colonisation with microorganisms as compared with normal fertile women. Contrary to our expectation, there was no statistically significant difference between infertile and pregnant women in the rate of cervical colonisation with the major pathogens. Candida albicans, Staphylococcus aureus and Neisseria gonorrheae were the most commonly isolated microorganisms; there was no difference between cases and controls in the rates of isolation of these pathogens.

There has been very little empirical research on the role of infection in infertility in Nigerian women. However, in the only available related study in the literature, Osoba et $a l^{11}$ found no difference between selected infertile and pregnant women in the rates of colonisation with the major sexually transmitted diseases in Ibadan, Nigeria. The rates of isolation of the microorganisms in this study appear high even for pregnant women but they conform to previously reported trends in this part of Nigeria. In published data, Rotimi and Somori ${ }^{12}$ reported a prevalence of Neisseria gonorrheae of $19.2 \%$ among 276 males and females in Lagos and Osoba ${ }^{13}$ reported an infection rate of $17.0 \%$ among hospitalised female patients. The infection rate for Candida albicans among pregnant women in Ibadan was $33 \% .{ }^{14}$ Similarly, Osoba et $a l^{15}$ reported an infection rate of $21 \%$ for Trichomonas vaginalis among pregnant women in Ibadan. Our findings of high infection rates in women in this study suggest a continuing high rate of sexually transmitted diseases in the area. This high prevalence of STDs in the community may partly explain the lack of a difference in the rates of infection between infertile and control patients since a wide segment of the community will thereby be implicated.

Our report suggests that women with secondary infertility have higher rates of infection with Neisseria gonorrheae, Candida albicans and Staphylococcus aureus than women with primary infertility. This could be due to the effect of age since women with secondary infertility were on average older than women with primary infertility; therefore a further case control study taking into account the effects of various confounding variables is needed to clarify this relationship. However, available reports indicate that most cases of secondary infertility in Africa are attributable to previous infection causing bilateral tubal occlusion and pelvic adhesions and up to $50 \%-80 \%$ of women with secondary infertility may have had prior infection. ${ }^{216}$ Many of the infections that cause tubal damage result from the ascent of gonorrhoea and possibly bacterial 
vaginosis organisms into the upper genital tract. Thus, it is conceivable that women with secondary infertility will have higher cervical carriage of Neisseria gonorrheae and other pathogenic organisms than women with primary infertility since some of the organisms could be carried for several years in the cervical crypts. In addition, it is possible that secondarily infertile women would be more susceptible to reacquisition with the same or another pathogenic organism since they are likely to continue with the sexual behaviour that put them at risk in the first place.

A major limitation of this study is the fact that it evaluates the cervix to identify current STD infection status in infertile and pregnant women. The differences in infection rate between the subgroups of subjects may simply reflect differences in current behaviour or practices between the groups. The results may have no association with previous experience of STDs which may be more important in establishing a causal link between STDs and infertility. However, we also found that more infertile women than pregnant women gave histories of previous induced abortions and pelvic inflammatory diseases requiring treatment which substantiates the role of infection as a major determinant of infertility in this group of women. A large prospective followup study will be required in a developing country with a high rate of STDs to establish clearly the relationship between high STD rates and infertility.

However, only $15 \cdot 2 \%$ of the infertile women gave a history of previous pelvic inflammatory disease despite the fact that close to $30 \%$ of the women had evidence of tubal occlusion at laparoscopy. This is not surprising since it is known that a large proportion of the pelvic infections that result in tubal occlusion are mild and often asymptomatic. ${ }^{17}$ This is not only true of gonococcal and vaginal candidosis-related infections but also of those infections that are caused by Chlamydia trachomatis, an organism that was not specifically looked for in this study. To date, no studies have specifically evaluated the association of Chlamydia trachomatis with infertility and tubal occlusion in Nigerian women. The prevalence of chlamydia infection in the general population is also not known. Such studies are clearly warranted to provide a basis for formulating comprehensive policies for addressing the problems of STDs and infertility in the country.

In this study, we found that the presence of vaginal discharge among infertile women did not accurately predict the cervical carriage of microorganisms. From a public health perspective, this implies that a policy of routinely screening women should be targeted at all infertile women and not simply confined to those with vaginal discharge or other symptoms of infection. With an STD isolation rate of close to $20 \%$, and an additional unknown proportion possessing Chlamydia trachomatis, it can be argued that such infertile women may benefit from a policy of routine antimicrobial treatment particularly in communities with no ready access to confirmatory laboratory tests. Such a policy will clearly not be justified in pregnant women because of the potential fetal toxicity of some antimicrobial agents.

The study showed that many of the isolated organisms in both groups of women were resistant to antibiotics that are commonly used in the area. In particular, Neisseria gonorrhoeae was resistant to ampicillin and spectinomycin and Staphylococcus aureus was resistant to ampicillin and gentamicin. This, no doubt, is due to intense antibiotic pressure occasioned by the widespread and inappropriate use of antibiotics in the area and the production of beta lactamases by the isolates. From this study, gentamicin and nalidixic acid appear to be the most effective in vitro antibiotics for Neisseria gonorrhoeae in this locality. Further work is warranted to determine the efficacy of the antibiotics within a clinical setting and the appropriate dosage regimen that would encourage patients' compliance.

In conclusion, women with secondary infertility in Nigeria have higher rates of cervical carriage of facultative and pathogenic microorganisms as compared with fertile pregnant controls.

A greater proportion of infertile women than pregnant women also gave historical evidence of previous pelvic inflammatory disease and induced abortion. These results suggest a causal role for pelvic infection in the high prevalence of secondary infertility in Nigerian women. We recommend that efforts to reduce the high rate of sexually transmitted diseases and lower genital tract infection in this population should be intensified. However, on a short term basis, women may benefit from a policy of routine screening for lower genital tract infections and STDs in all family planning and antenatal clinics. In areas where laboratory services are limited, infertile women could be routinely treated with antimicrobial agents which are identified as being effective against local strains of organisms.

We are grateful to Drs Abejide and Fasubaa for providing clinical assistance at the infertility and antenatal clinics. The study was conducted with funds provided by the Obafemi Awolowo University Research Committee grant, number $1427 \mathrm{CT}$.

1 Adetoro O, Ebomoyi EW. Prevalence of infertility in a rural Nigerian community. African fournal of Medicine rural Nigerian community. African

2 World Health Organization. Infections, pregnancies and infertility: perspectives on prevention. Fertil Steril 1987 47:944-9.

3 Otolorin EO, Ojengbede O, Falase AO. Laparoscopic evaluation of the tuboperitoneal factor in infertile Nigerian women. Int $\mathcal{f}$ Gynaecol Obstet 1987;25:47-52.

4 Okonofua FE, Esen UI, Nimalaraj T. Hysterosalpingography versus laparoscopy in tubal infertility. Comparison based on findings at laparotomy. Int Gynaecol Obstet 1989;28:143-7.

5 Otubu JA, Sagay AS, Dauda S. Hysterosalpingogram, laparoscopy and hysteroscopy in the assessment of the infertile Nigerian female. $E$ Afr Med $\mathcal{F} 1990 ; 67: 370-4$.

6 Adekunle AO, Ladipo OO. Reproductive tract infections in Nigeria: challenges for a fragile health infrastructure. In: Germain A, Holmes KK, Pilot P, Wasserheit JN. eds. Reproductive Tract Infections. Plenum Press, New eds. Reproductive Tra

7 Okonofua FE, Makinde ON, Ayangade SO. Yearly trends in caesarean section and caesarean mortality at Ile-Ife, Nigeria. Trop $\mathcal{f}$ Obstet Gynaecol 1988;1:31-5.

8 Okonofua FE, Onwudiegwu U, Odunsi AO. Illegal induced abortion: a study of 74 cases in Ile-Ife, Nigeria Tropical Doctor 1992;22:75-8. 
9 Ibeziako PA. Effect of post-caesarean section sepsis on subsequent fertility. W Afr f Med 1986;5:35-9.

10 World Fertility Survey. The Nigerian Fertility Survey, 1981-82: A summary of findings, Voorburg. The Netherlands, International Statistical Institute; 1984.

11 Osoba AO, Onifade A, Alausa KO. Genital tract infection and reproductive failure in developing countries. Nig $\mathcal{f}$ Med 1975;5:401-6.

12 Rotimi VO, Onifade A. Sexually transmitted diseases in clinic patients in Lagos. $B r F$ Venereal Dis 1980;56:54-6.

13 Osoba AO. Epidemiology of urethritis in Ibadan. $\mathrm{Br} f$ Venereal Dis 1972;48:116-20.

14 Onifade A, Osoba AO. Venereal diseases among women complaining of infertility. W Afr f Med 1975;23:284-6. 5 Osoba AO, Onifade A. Venereal disease among pregnan women in Ibadan, Nigeria. W Afr $\mathcal{F}$ Med 1973;1:39-41.

16 Westrom L, Mardl PA. Acute pelvic inflammatory disease. In Sexually Transmitted Diseases: Holmes KK, Mardh P, Sparling PF, Weismer PJ, Cates W, Lemon SM, Staman WE eds. New York: McGraw-Hill 1989:593615.

17 De Muylder X, Laga $M$, Tennstedt C, Van Dyck E Aelbers GN, Piot P. The role of Neisseria gonorrhoea and Chlamydia trachomatis in pelvic inflammatory disease and its sequelae in Zimbabwe. $\mathcal{F}$ Infect Dis 1990; 162:501-5 